University of Nebraska - Lincoln

DigitalCommons@University of Nebraska - Lincoln

$5-15-2009$

\title{
Selective Nanoshaving of Self-Assembled Monolayers of 2-(4-Pyridylethyl)Triethoxysilane
}

\author{
Luis G. Rosa \\ University of Nebraska-Lincoln, luis.rosa13@upr.edu \\ Jinyue Jiang \\ University of Nebraska-Lincoln, jjiang2@unl.edu \\ Ocelio V. Lima \\ University of Nebraska-Lincoln \\ Jie Xiao \\ University of Nebraska-Lincoln, jie.xiao@helmholtz-berlin.de \\ Emmanuel Utreras \\ University of Nebraska-Lincoln \\ See next page for additional authors
}

Follow this and additional works at: https://digitalcommons.unl.edu/physicsdowben

Part of the Physics Commons

Rosa, Luis G.; Jiang, Jinyue; Lima, Ocelio V.; Xiao, Jie; Utreras, Emmanuel; Dowben, Peter A.; and Tan, Li, "Selective Nanoshaving of Self-Assembled Monolayers of 2-(4-Pyridylethyl)Triethoxysilane" (2009). Peter Dowben Publications. 222.

https://digitalcommons.unl.edu/physicsdowben/222

This Article is brought to you for free and open access by the Research Papers in Physics and Astronomy at DigitalCommons@University of Nebraska - Lincoln. It has been accepted for inclusion in Peter Dowben Publications by an authorized administrator of DigitalCommons@University of Nebraska - Lincoln. 


\section{Authors}

Luis G. Rosa, Jinyue Jiang, Ocelio V. Lima, Jie Xiao, Emmanuel Utreras, Peter A. Dowben, and Li Tan 


\title{
Selective nanoshaving of self-assembled monolayers of 2-(4-pyridylethyl)triethoxysilane
}

\author{
Luis G. Rosa, ${ }^{\mathrm{a}, \mathrm{b}, \mathrm{c}}$ Jinyue Jiang, ${ }^{\mathrm{d}}$ Ocelio V. Lima, ${ }^{\mathrm{d}}$ Jie Xiao, $^{\mathrm{a}}$ Emmanuel Utreras, ${ }^{\mathrm{a}, \mathrm{b}, \mathrm{c}}$ \\ P. A. Dowben, ${ }^{\mathrm{a}}$ and Li Tan ${ }^{\mathrm{d}}$ \\ a Department of Physics and Astronomy and the Nebraska Center for Materials and Nanoscience, \\ University of Nebraska-Lincoln, P.O. Box 880111, Lincoln, NE 68588-0111, USA \\ ${ }^{\mathrm{b}}$ Department of Physics and Electronics, University of Puerto Rico-Humacao, \\ 100 Road\#908, CUH Station, Humacao, 00791, Puerto Rico \\ ${ }^{\mathrm{c}}$ Institute for Functional Nanomaterials, University of Puerto Rico-Rio Piedras, Rio Piedras, PR 00931, USA
}

${ }^{\mathrm{d}}$ Department of Engineering Mechanics, W306 Nebraska Hall, University of Nebraska-Lincoln, Lincoln, NE 68588-0526, USA

Corresponding author - P. A. Dowben, tel 402 472-9838, email pdowben@unl.edu

\begin{abstract}
Single molecular layers of 2-(4-pyridylethyl)triethoxysilane have been deposited on native oxide surfaces of silicon, with the triethoxysilylethyl groups towards the silicon oxide interface and pyridine at the surface. It is possible to "shave" or mechanically break the molecular bonds at the alkoxy-silane ( $\mathrm{Si}-\mathrm{C}$ ) bond using scanning atomic force microscope, leaving large swaths of surface area cut to a depth of $0.64 \pm 0.06 \mathrm{~nm}$, exposing the silicon of the alkoxy-silane ligand. Mechanical cleavage of the pyridine ligand alone is also possible, but more difficult to control selectively.
\end{abstract}

Keywords: nanoshaving, self-assembled monolayers, 2-(4-pyridylethyl)triethoxysilane, atomic force microscope, electronic structure of organic layers

\section{Introduction}

While selective bond breaking of a variety of chemical species is known and has been demonstrated, the mechanical breaking of specific chemical bonds in an adsorbed species is more of a challenge. Nanometer scale lithographies, using an atomic force microscope (AFM) tip have been successfully demonstrated in the removal of material [1-7, often a self-assembled organic layer. Such examples are sometimes accompanied by replacement with another organic species [1, 2, 8-11]. The former type of processing is nanoshaving [1-7] and the latter is "dip pen" nanolithography or "nanografting" [1, 2, 8-11]. Selective oxidation of a self-assembled monolayer is also possible [2]. While this type of nanoshaving can lead to well defined "nanoscale" features in a monolayer molecular film [1-11], selective mechanical "cutting" or bond breaking within the molecule, for only part of the molecular film, has not been demonstrated. Over-cutting or etching using an AFM tip is certainly possible [3$7,12]$, but mechanically engineering the organic surface selectively within the monomolecular layer opens up new vistas for selective nanoscale surface modifications and surface chemistry.
Selective mechanical bond breaking of an adsorbate, using an atomic force microscope, has several requirements: an adsorbate well anchored to the substrate and a rigid molecular backbone with at least one fairly "weak" bond where the AFM tip can "shear" off part of the molecule. In addition, if the cleaved fragment is either volatile or "sticky", then the shaved area can be prepared largely free of fragment debris. The complications that need to be avoided in the selective mechanical "shearing" process are the removal or displacement of all of the adsorbate species (as opposed to shaving off a portion of the molecule), or the mechanical canting or "tipping" the orientation of the adsorbate species. Layers made from alkoxy silane species, with various end group ligands, have unique merits as these molecular complexes form strong bonds with an oxide surface and can be used to modify both the dielectric properties and the optical properties of the surface [13]. In this paper we focus on 2-(4-pyr idylethyl)triethoxysilane molecules, schematically shown in Figure 1, to demonstrate a selective mechanical cutting of molecular layers on $\mathrm{SiO}_{2}$. 

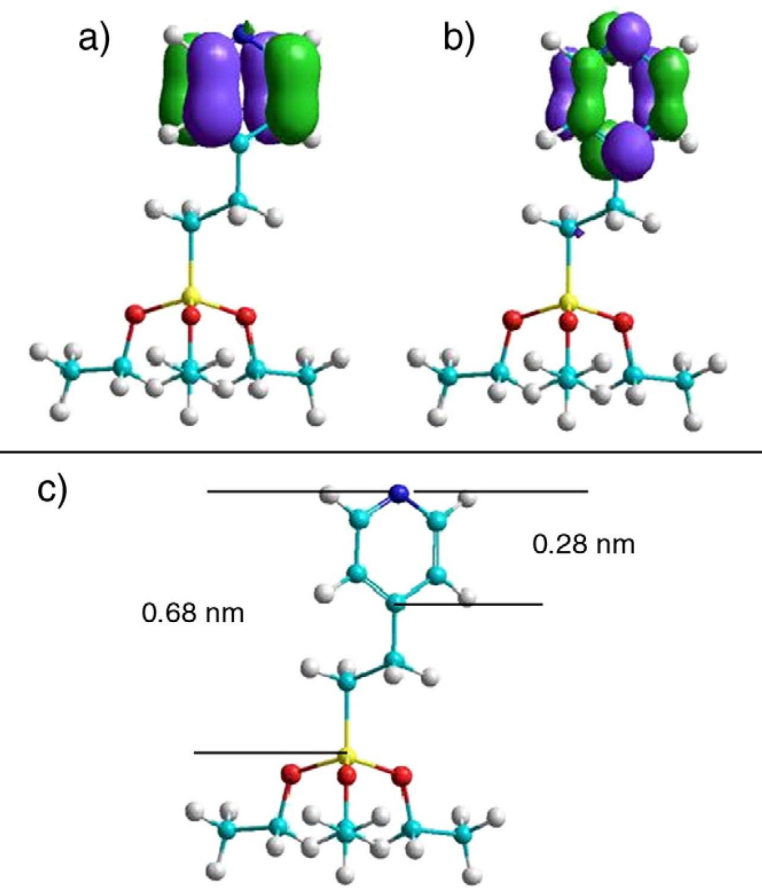

Figure 1. The structure of 2-(4-pyridylethyl)triethoxysilane. The HOMO-LUMO band gap is dominated by states originating from pyridine terminal of the 2-(4-pyridylethyl)triethoxysilane molecule as indicated by the HOMO (a) and LUMO (b). The calculated structure and vertical distance along the surface normal for 2-(4-pyridylethyl)trietho xysilane adsorbed on silicon oxide (c). Worth noting are the width of pyridine at $0.28 \mathrm{~nm}$ and vertical distance of $0.68 \mathrm{~nm}$ between $\mathrm{N}$ of the pyridine and the Si.

\section{Experimental}

The polished p-type $\operatorname{Si}(100)$ wafers were pre-cleaned to remove organic contamination and particles and immersed in piranha solution $\left(\mathrm{H}_{2} \mathrm{SO}_{4}: \mathrm{H}_{2} \mathrm{O}_{2}-1: 1\right.$ by volume $)$ to hydroxylate the silicon wafer surfaces, making them extremely hydrophilic. The native oxide surface was untouched by this procedure. The hydrophilic wafers were then immersed in a toluene solution of 2-(4-pyridylethyl)trieth oxysilane (Gelest), and the excess molecules were removed by rinsing and sonicating in a fresh toluene solvent. After repetition, the wafers were annealed at $50{ }^{\circ} \mathrm{C}$ in high humidity $(\sim 80 \%$ relative humidity) for $8 \mathrm{~h}$ and immersed once more in the same silane solution and rinsed and sonicated and then finally baked at $115^{\circ} \mathrm{C}$. The latter step aims to convert the hydrogen bonds of the adsorbed 2-(4pyridylethyl)triethoxysilane to the silicon oxide surface to covalent bonds. The process from the first immersion until baking was repeated twice more leading to a more complete filling of the monolayer molecular film, as characterized in AFM.

Nanostructuring and imaging were both carried out using a Digital Instruments AFM with a Nanoscope IIIa controller. After one complete deposition cycle, as described above, the 2-(4-p yridylethyl)triethoxysilane film thickness was measured to be 0.9 to $1.1 \mathrm{~nm}$ from atomic force microscope (AFM) height profiles. A film thickness of $1.0 \pm 0.1 \mathrm{~nm}$ was also determined from variable angle ellipsometry of a complete 2-(4-pyridylethyl)triethoxysilan e monomolecular films ( 3 cycles as described above). These two measurements make it clear, after taking into account the molecular geometrical considerations (Figure 1), that the long molecular axis is roughly parallel with the silicon oxide surface normal.

Combined photoemission (UPS) and inverse photoemission (IPES) spectra were taken for different coverages of 2-(4-pyridyl ethyl)triethoxysilane deposited on the silicon oxide substrates, for coverages nominally up to one monolayer. Both photoemis- sion and inverse photoemission spectroscopy measurements were made in the same ultrahigh vacuum (UHV) chamber [14-16]. The photoemission spectra were taken with a hemispherical electron energy analyzer using $\mathrm{He}$ I radiation of $21.2 \mathrm{eV}$, with the photoelectrons collected along the surface normal. The inverse photoemission spectra (IPES) were obtained by using electrons with variable incident energy while measuring the emitted photons at a fixed energy $(9.7 \mathrm{eV})$ using a Geiger-Müller detector (isochromat mode), as described elsewhere [7-9]. In both photoemission and inverse photoemission measurements, the binding energies are referenced with respect to the Fermi edge of UHV chamber, in contact with the sample surface.

Measurements were taken of the self-assembled monolayer at substrate temperatures of $150 \mathrm{~K}$ (and below). There are strong indications of either decomposition or desorption of the 2-(4-pyrid ylethyl)triethoxysilane during inverse photoemission experiments at substrate temperatures of $300 \mathrm{~K}$ and above. The lower temperatures in the region of $150 \mathrm{~K}$ were found to suppress electron stimulated desorption and/or decomposition and thermal desorption of 2-(4-pyridylethyl)triethoxysilane during the measurements.

\section{Electronic structure}

The combined ultraviolet photoemission spectroscopy (UPS) and inverse photoemission spectroscopy (IPES) measurements provided an indication of the molecular orbital placement of both occupied and unoccupied orbitals of 2-(4-pyridylethyl)triethoxysilan $\mathrm{e}$, as indicated in Figure 2. With repeated cycles of 2-(4-pyridyleth yl)triethoxysilane exposure to the silicon oxide, as described above, the highest occupied molecular orbital (HOMO) to lowest unoccupied molecular orbital (LUMO) gap is seen to increase and features associated with the 2-(4-pyridylethyl)triethoxysilane molecular orbitals are evident.

Indeed, the results from the combined ultraviolet photoemission spectroscopy and inverse photoemission spectroscopy of

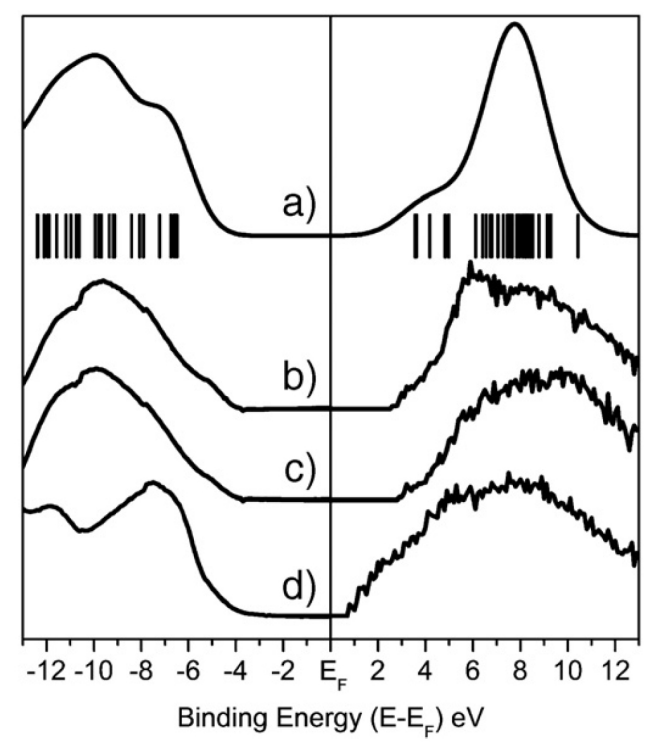

Figure 2. The electronic structure of 2-(4-pyridylethyl)triethoxysilane from combined photoemission and inverse photoemission spectroscopy, as a function of 2-(4-pyridylethyl)triethoxysilane coverage. The clean silicon oxide $\left(\mathrm{SiO}_{2}\right)$ substrate formed on p-doped silicon (d) can be compared to the half surface coverage (1 cycle) of 2-(4-pyridylethyl)trie thoxysilane (c) and the full surface coverage (3 cycles) of 2-(4-pyridylet hyl)triethoxysilane (b). The experimental electronic structure (b) is also compared with the calculated density of states based on a single isolated molecule (a). The theoretical Eigenvalues are shown below the expected density of states, uncorrected for solid state and matrix element effects. 

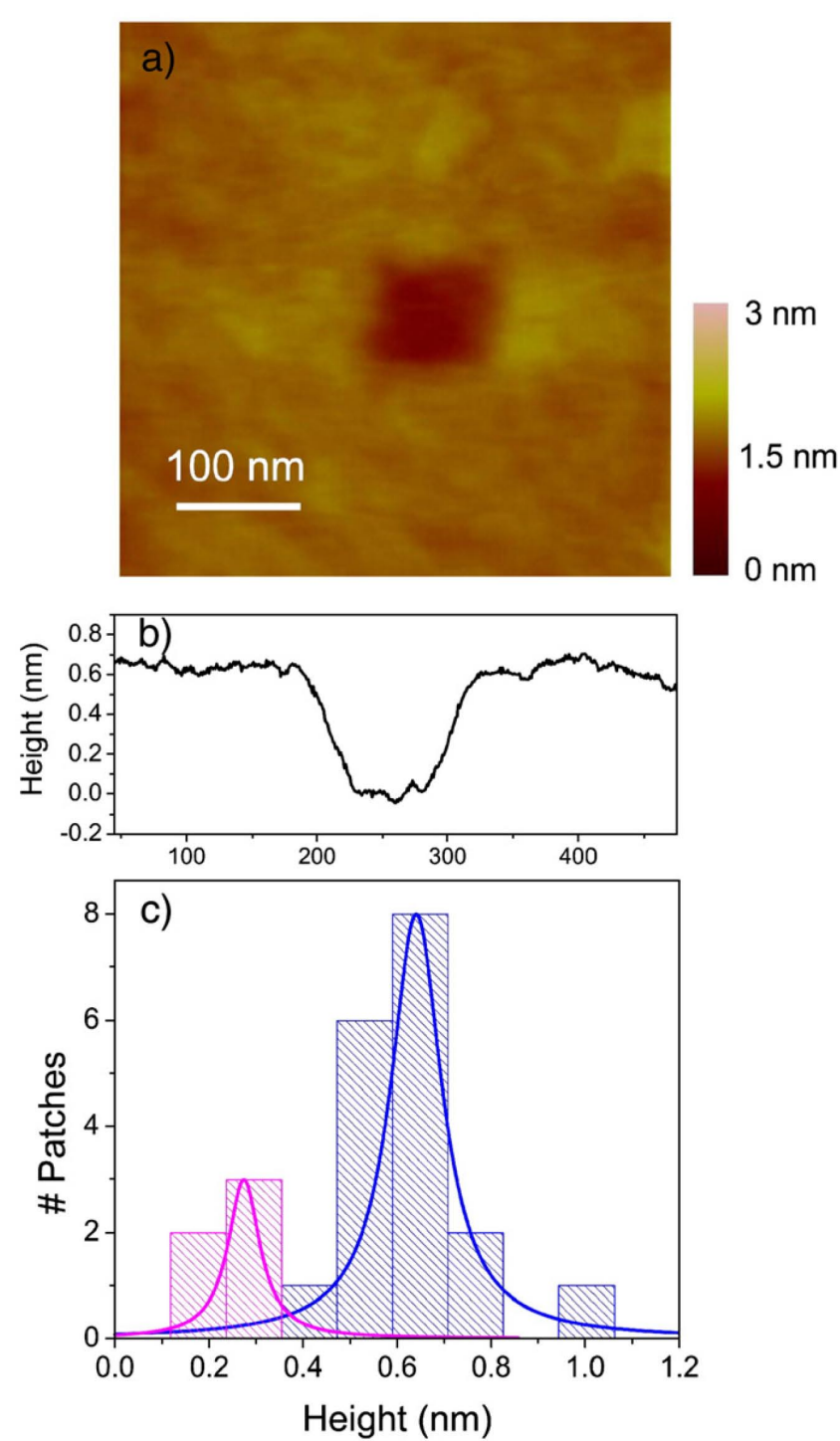

Figure 3. Nanoshaving of 2-(4-pyridylethyl)triethoxysilane monomolecular films. An AFM image of a $100 \mathrm{~nm} \times 100 \mathrm{~nm}$ nanoshaved region of 2-(4-pyridylethyl)triethoxysilane is shown in (a), with the height profile or cross-section of nanoshaved area or patch indicated in (b). A compilation AFM nanoshaving experiments for 2-(4-pyridylethyl )triethoxysilane thin films, as a histogram of the cut depth measurements taken from height profiles (c). The histogram was fitted with two Gaussians with peak centers at $0.28 \mathrm{~nm}$ and $0.64 \mathrm{~nm}$, as indicated in panel (c).

2-(4-pyridylethyl)triethoxysilane self-assembled on $\mathrm{SiO}_{2}$ substrates are consistent with expectations, if the molecule is aligned with the long molecular axis along the surface normal. As seen in Figure 2c, the highest occupied molecular orbital (HOMO) to lowest unoccupied molecular orbital (LUMO) gap for 2-(4-pyridylethyl)triethox ysilane is somewhat smaller (about $8.5 \mathrm{eV}$ ) than expected (which would be about $10 \mathrm{eV}$ ) from semi-empirical method NDO-PM3 (neglect of differential diatomic overlap, parametric model number 3) model calculations based on Hartree-Fock formalism [17]. Although PM3 is a simplistic semiempirical calculation, density functional theory (DFT) is notorious for underestimating the band gap sometimes by a factor 2 or more [18], particularly for molecular systems, and must be rescaled for comparison with experiment [19], particularly final state spectroscopies like photoemission and inverse photoemission. To compare the model calculations with experiment (Figure 2), we applied Gaussian envelopes of $1.8 \mathrm{eV}$ full width half maximum to each calculated molecular orbital energy (Eigenvalue) to account for the solid state broadening in photoemission and then summing. This model density of state calculations is rigidly shifted in energy by $3.4 \mathrm{eV}$ and then compared with the combined photoemission and inverse photoemission data, as indicated in Figure 2. The $3.4 \mathrm{eV}$ energy shift which is, for the most part but not completely, representative of work function $\Phi$ equal to the difference of vacuum energy $E_{\mathrm{vac}}$ and Fermi level $E_{\mathrm{F}}$, is applied to the calculated electronic structure uniformly. No corrections were made for final state effects or matrix element effects in either calculation, so the comparison with experiment is simplistic, but nonetheless still often successful [14, 20,21].

The intermolecular interactions within the 2-(4-pyridylethyl)t riethoxysilane molecular film and the strong molecular (covalent bond) interaction with the silicon oxide substrate (i.e. solid state effects [22]) are the likely origin of the differences between the experimental HOMO-LUMO gap, based on the combined photoemission and inverse photoemission, and expectations based on a single molecule model semi-empirical calculations.

The experimental inverse photoemission is dominated by the pyridine molecular orbitals closer to the Fermi level, and thus differs significantly from the model calculations, which are uncorrected for matrix element effects and molecular orientation. The greater intensity of these pyridine weighted molecular orbitals is consistent with an "upright" molecular orientation. Such strong dependence of the inverse photoemission spectra on molecular orientation has been seen for copper phthalocyanine (CuPc) [7] and polyphenyl thiols [20], and tends to be more significant than in the case of photoemission [7], as is observed here. The proximity of the pyridine molecular orbitals to the Fermi level will tend to make this ligand "sticky" and enhance possible adherence to the AFM tip.

\section{Nanoshaving the SAM layer to different depths}

The process of nanoshaving has been described extensively elsewhere [1-7]. Here, an AFM tip was used with a SiN cantilever and a $0.58 \mathrm{~N} / \mathrm{m}$ spring constant so that in the low force contact mode the surface morphology could be imaged (Figure 3a). To perform nanoshaving, load forces of $50 \mathrm{nN}$ to $110 \mathrm{nN}$ were applied by the tip to the SAM layer surface. In this force range, a complete scraping off of the 2-(4-pyridylethyl)triethoxysilane molecular film was not observed, rather nanoshaving cut depths of $0.64 \mathrm{~nm}$ were seen and to a lesser extent cut depths of $0.27 \mathrm{~nm}$. As illustrated in the AFM image in Figure 3, a $100 \mathrm{~nm} \times 100 \mathrm{~nm}$ nanoshaved region of 2-(4-pyridylethyl)triethoxysilane film can be imaged showing sharp boundaries. The height profile or crosssection of nanoshaved area or patch indicated (Figure $3 b$ ) provides no indication of molecular canting induced mechanically.

The compilation AFM nanoshaving experiments (Figure 3c) for 2-(4-pyridylethyl)triethoxysilane thin films suggest that there is selective mechanical cleavage of the 2-(4-pyridylethyl)triethoxy silane molecular film at $0.64+0.06 \mathrm{~nm}$ depth (Figure 3c) or at the $\mathrm{Si}-\mathrm{C}$ bond as indicated in Figure 1, with a minority of nanoshaving experiments of a cut depth of $0.28+0.05 \mathrm{~nm}$. At $50 \mathrm{nN}$ applied force, cut depths were observed to all be near the value of $0.28 \mathrm{~nm}$. At $100 \mathrm{nN}$ applied force the preponderance of nanoshaving cut depths observed was at $0.68 \mathrm{~nm}$.

The bond energies at the pyridine to ethyl "neck" at a depth of $0.28 \mathrm{~nm}$ and at the siloxy-ethyl bond at an expected depth of $0.68 \mathrm{~nm}$ are relatively weak at 71 and $76 \mathrm{Kcal} / \mathrm{mol}$ [23], so these are both likely mechanical cleavage points of a molecule oriented with long molecular axis perpendicular to the shear direction (as is the case here). Since the molecular orbitals of the pyridine end group dominate the highest and lowest molecular orbitals, the pyridine end group should dominate the interaction with the AFM probe tip, thus aiding the mechanical nanoshaving process. 


\section{Summary}

What is clear is that chemically selective nanoshaving can be achieved, with the correct choice of an anchor end group to the substrate, linking groups and well chosen applied force to the AFM tip. We do not at present have an accurate assessment of the shear forces needed for this selective mechanical bond breaking and this remains a matter of some interest.

\section{Acknowledgments}

This research was supported by the National Science Foundation through grant number CHE-0415421, CHE-0650453 and CMMI 0825905, the Army Research Office (W911NF-08-1-0190) and the NSF “QSPINS” MRSEC (DMR 0213808).

\section{References}

[1] M.E. Greene, C.R. Kinser, D.E. Kramer, L.S.C. Pingree and M.C. Hersam, Microsc Res Technique 64 (2004), p. 415.

[2] R. Garcia, R.V. Martinez and J. Martinez, Chem Soc Rev 35 (2006), p. 29.

[3] J. Shi, J. Chen and P.S. Cremer, J Am Chem Soc 130 (2008), p. 2718.

[4] G.-Y. Liu, S. Xu and Y.L. Qian, Acc Chem Res 33 (2000), p. 457.

[5] M. Kaholek, W.K. Lee, B. LaMattina, K.C. Caster and S. Zauscher, Nano Lett 4 (2004), p. 373.

[6] N.A. Amro, S. Xu and G.-Y. Liu, Langmuir 16 (2000), p. 3006.

[7] J. Lian, L.G. Rosa and G. Scoles, J Phys Chem C 111 (2007), p. 17275.

[8] M. Jaschke and H.J. Butt, Langmuir 11 (1995), p. 1061.
[9] R.D. Piner, J. Zhu, F. Xu, S.H. Hong and C.A. Mirkin, Science 283 (1999), p. 661

[10] K.B. Lee, S.J. Park, C.A. Mirkin, J.C. Smith and M. Mrksich, Science 295 (2002), p. 1702.

[11] J. Hyun, S.J. Ahn, W.K. Lee, A. Chilkoti and S. Zauscher, Nano Lett 2 (2002), p. 1203.

[12] J.E. Headrick, M. Armstrong, J. Cratty, S. Hammond, B. Sheriff and C.L. Berrie, Langmuir 21 (2005), p. 4117.

[13] J.J. Senkevich, C.J. Mitchell, G.-R. Yang and T.-M. Lu, Langmuir 18 (2002), p. 1587.

[14] J. Xiao, A. Sokolov and P.A. Dowben, Appl Phys Lett 90 (2007), p. 242907.

[15] B. Xu, J. Choi, A.N. Caruso and P.A. Dowben, Appl Phys Lett 80 (2002), p. 4342.

[16] D.N. McIlroy, C. Waldfried, T. McAvoy, J. Choi, P.A. Dowben and D. Heskett, Chem Phys Lett 264 (1997), p. 168.

[17] J.J.P. Stewart, "PM3" in Encyclopedia of Computational Chemistry, Wiley (1998).

[18] I.N. Yakovkin and P.A. Dowben, Surf Rev Lett 14 (2007), p. 481.

[19] A.M. Scheer and P.D. Burrow, J Phys Chem B 110 (2006), p. 17751.

[20] D.-Q. Feng, D. Wisbey, Ya B. Losovyj, Y. Tai, M. Zharnikov and P.A. Dowben, Phys Rev B 74 (2006), p. 165425.

[21] J. Xiao, L.G. Rosa, M. Poulsen, D.-Q. Feng, S. Reddy, J.M. Takacs, L. Cai, J. Zhang, S. Ducharme and P.A. Dowben, J Phys Cond Matter 18 (2006), p. L155.

[22] P.A. Dowben, Jaewu Choi, E. Morikawa and Bo Xu, Handbook of thin films. In: H.S. Nalwa, Editor, Characterization and Spectroscopy of Thin Films, Chapter 2 vol. 2, Academic Press (2002), pp. 61-114.

[23] R.T. Sanderson, Chemical Bonds and Bond Energy (2nd ed.), Academic Press Inc (1976) ISBN-10:0126180601. 